\title{
Integrated Trajectory Planning and Torque Vectoring for Autonomous Emergency Collision Avoidance
}

\author{
Shayan Taherian, Umberto Montanaro, Shilp Dixit and Saber Fallah
}

\begin{abstract}
This paper proposes an integrated trajectory planning based on Model Predictive Control (MPC) for designing collision-free evasive trajectory and a torque vectoring controller based on optimal control to ensure lateral-yaw stabilization in autonomous emergency collision avoidance under low friction and crosswinds on highways. The trajectory for performing the evasive manoeuvre is designed to minimise the deviation of the vehicle from the lane center while ensuring the vehicle remains within the road boundaries. The steering input computed from the MPC is used to steer the vehicle along the reference trajectory while the torque vectoring controller provides additional lateral-yaw stability. The integrated control framework was implemented on IPG CarmakerMATLAB co-simulation platform and its efficacy was evaluated under different scenarios. Simulations performed for emergency collision avoidance at high speeds with low road friction and heavy crosswinds confirm the ability of the proposed closed-loop framework at successfully avoiding collisions with moving obstacles while ensuring that the controlled vehicle remains within its limits of stability. Furthermore, the robustness of the proposed control framework to variations in road friction changes is demonstrated by simulating an evasive manoeuvre at high-speeds for wide range of road friction conditions. Comparing the performance of the proposed control framework to a vehicle without the corrective actions available via torque vectoring highlight the additional benefits provided by the proposed closed-loop scheme at ensuring lateral-yaw stability under emergency scenarios.
\end{abstract}

\section{INTRODUCTION}

Over the last few years there has been an increasing interest in autonomous driving as a possible solution to improve road safety, passengers comfort, and fuel consumption [1]. Hence, researchers and practitioners are focusing on the design and implementation of autonomous driving functionalities to allow autonomous vehicles to plan and perform common manoeuvres in urban and highway scenarios, such as overtaking [2], crossing intersections [3], lane change [4] and etc in a safe and efficient manner. In addition to the development of such functionalities for autonomous driving, it is important for autonomous vehicles to manage emergency situations caused by sudden changes in motion of surrounding vehicles such as harsh braking of a preceding vehicle [5]. Therefore, there is a need of developing intelligent collision avoidance systems to prevent catastrophic accidents. Typically, a collision avoidance system of an autonomous vehicle is

\footnotetext{
${ }^{1}$ Shayan taherian,Umberto Montanaro,Shilp Dixit and Saber Fallah are with Faculty of Mechanical Engineering, University of Surrey, Guildford, GU2 7XH, UK, s.fallah@surrey.ac.uk
}

responsible for generating and executing a collision free path while maintaining the stability of the vehicle. For instance, in [6] a collision avoidance system was developed using polynomial parametrization method as trajectory planer and a MPC as the path tracker of the system. The resulting controller was capable of creating and tracking collision free trajectories in the presence of stationary and moving obstacles. Shah et al. [7] proposed a hierarchical scheme including threat assessment, path planning and path tracking using state feedback controller. The proposed approach showed the enhancement of the vehicle stability under adverse driving conditions. Gao et al [8] developed two levels of MPC framework for trajectory planning and tracking controller for obstacle avoidance in autonomous vehicles on slippery roads. Although the control structure was validated experimentally in low surface condition at the velocity of $55 \mathrm{~km} / \mathrm{h}$, the results were limited to stationary obstacles. Cao et al [9] proposed a hierarchical MPC method for autonomous emergency collision avoidance scenario. In this work, trajectory planning was designed based on artificial potential field and nonlinear MPC to generate a feasible trajectory and tracking control using linear time varying (LTV) MPC. The proposed approach was capable of tracking collision free trajectory and maintain vehicle stability in an evasive maneuver. Funke et al [10] proposed a control strategy for path tracking and vehicle stabilization using MPC in collision avoidance scenario. This approach was tested experimentally on an autonomous vehicle and demonstrated its capability of avoiding accident in emergency collision avoidance scenario on a curve. Although, the above designs were successful to avoid collision in certain conditions, the control performance in the presence of external disturbances, sudden variation of the preceding vehicle motion or adverse road conditions were only marginally investigated. It is noted that such external disturbances can jeopardize the tracking performance of the system and even induce vehicle instability. For instance, vehicle's braking operation under low surface friction causes the tires to be locked, leading to vehicle instability. As another example, strong crosswinds can veer off the vehicle from the road boundary especially at high speed, causing the vehicle to lose its directional stability. A possible solution for maintaining stability is through the use of torque vectoring control which can significantly improve vehicle response in steady-state 


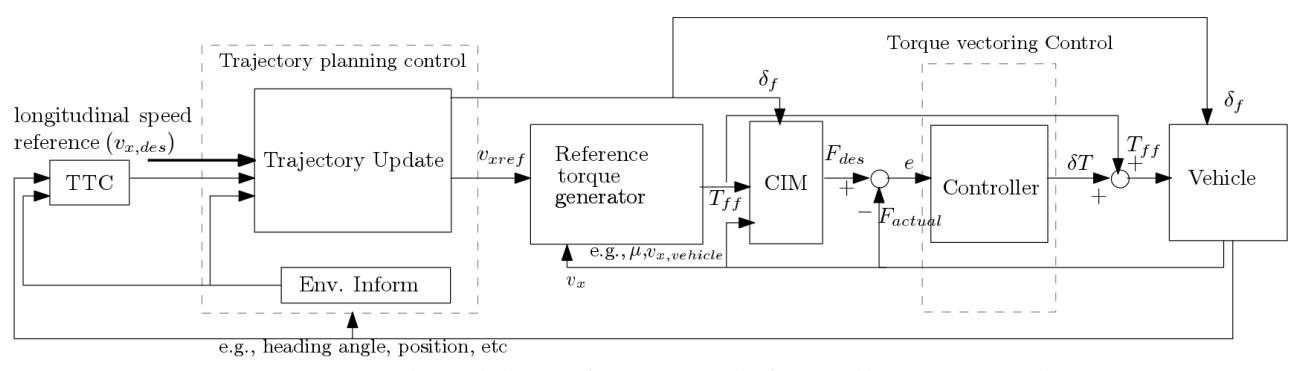

Fig. 1: Closed-loop framework for collision avoidance system

and transient driving conditions [11]. The concept of torque vectoring is to control the traction and braking torque of each wheel to generate a direct yaw moment. In order to benefit from advantages of torque vectoring controllers in collision avoidance system applications of autonomous vehicles in emergency scenarios [12][13], this paper proposes a novel control architecture where MPC is used to design feasible and safe trajectories for emergency collision avoidance and a torque vectoring controller to ensure that the vehicle can follow the designed reference without any loss of yaw-lateral stability. The results show that the proposed control strategy can maintain autonomous vehicle's stability while avoiding collision in high-speed and low surface friction conditions under presence of external disturbances such as heavy crosswind. The trajectory planning controller presented in this paper is an extension of the work done in [14]. The main difference lies in the use of a bicycle model of the vehicle instead of a point mass model as used in [14] since a bicycle model captures vehicle dynamics more accurately which is a crucial requirement for computing admissible trajectories for collision avoidance. The stabilization of the vehicle motion during the emergency maneuver is based on the torque-vectoring technique proposed in [15]. In accordance to [15], this approach allows the vehicle to be manipulated as nominal driving conditions (e.g., high friction surface) even in the presence of uncertainties and external disturbances. The efficacy of the integrated trajectory planning and the stabilization controller is demonstrated for emergency collision avoidance in high-speed and low friction environments. Moreover, the robustness of such integrated control scheme has been tested in the presence of heavy cross wind and has been validated in IPG CarMakerSimulink co-simulation environment. The remaining of the paper is structured as follows: Section II introduces control system architecture in the paper. In section III. The control formulation for trajectory planning, torque vectoring and reference torque generator will be discussed. In section IV] the numerical results will be examined. At the end, the concluding remarks are discussed in Section $\mathrm{V}$

\section{CONTROL SYSTEM ARCHITECTURE}

The architecture of the proposed integrated control structure is shown in Fig. 1. which consists of trajectory planner, reference torque generator, Command Interpreter Module (CIM) and torque vectoring controller. The trajectory planner uses the information from environment and generates trajectories of longitudinal speed, $v_{x, \text { ref }}$ and steering angle, $\delta_{f}$. The reference torque generator block is responsible for adjusting braking and traction torques such that the deviation between the desired and actual vehicle speeds is minimized. The CIM generates desired forces at the Center of Gravity (C.G.) based on the inputs from the trajectory planner under the normal driving conditions. CIM continuously monitors the inputs from the trajectory planner as well as the states of the vehicle to provide accurate desired values to the torque vectoring controller to keep the vehicle within the stable region. In this paper, CIM has been derived using a four-wheels vehicle model in [16] which includes magic formula as tire model [17]. The torque vectoring controller minimizes the error between the desired C.G. forces $\left(F_{\text {des }}\right)$, generated from CIM and actual C.G forces $\left(F_{\text {actual }}\right)$ obtained from the vehicle and consequently generates corrective braking/traction torques for each wheel in order to adjust the nominal torques applied by the reference torque generator. Furthermore, it is assumed the trajectory planning controller for generating the optimal steering is activated when the time to collision (TTC) is $\leq 2$ s. It is noteworthy that current on-board emergency systems are activated when the TTC value goes below 3s [18]. Thus, in this paper the ability of the proposed collision avoidance system will be assessed under more challenging conditions.

\section{CONTROL FORMULATION}

\section{A. Trajectory planning}

For solving the collision avoidance problem, a path must be planned for the subject vehicle so as (i) to maintain the vehicle position at the centre line of the desired lane, $y_{d e s}$, (ii) to keep the vehicle at the preferred velocity while avoiding collision from the preceding vehicle, (iii) and to ensure the vehicle stays within the road boundary. For the trajectory planning using MPC, a kinematic bicycle vehicle model is used. 


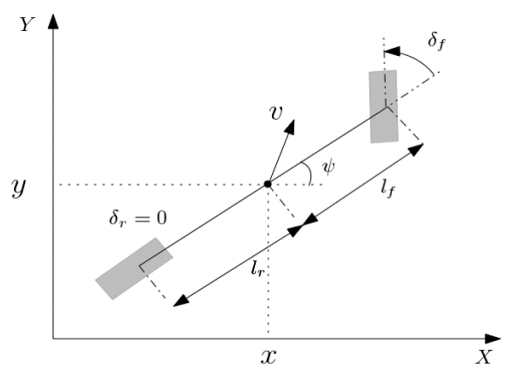

Fig. 2: Kinematic bicycle model

The dynamics of the subject vehicle in a road aligned coordinate frame represented as linear kinematic bicycle model [19] is demonstrated in Fig. 2.

In this model, the states of the subject vehicle are denoted as $\xi \triangleq\left[x, y, v_{x}, \psi\right]^{T}$ where $x$ and $y$ are the longitudinal and lateral position in inertial frame $(X, Y), \psi$ is the inertial heading angle, and $v_{x}$ is the longitudinal velocity of subject vehicle. $l_{f}$ and $l_{r}$ represent the distance from the center of the gravity of the vehicle to the front and rear axles, respectively. Consider the discretized linear time-invariant system dynamics represented in a state-space format given below.

$$
\xi_{t+1}=A \xi_{t}+B u_{t}
$$

where $u \triangleq\left[\delta_{f}, a_{x}\right]^{T}$ with $\delta_{f}, a_{x}$ being the steering angle and the longitudinal acceleration respectively. Furthermore, the system matrices $A$ and $B$ are state and input matrices as reported in [2]. System (1) is subjected to the following state and input constraints:

$$
\xi \in \hat{\mathcal{X}}, u \in \hat{\mathcal{U}}
$$

where $\hat{\mathcal{X}}=\left\{\xi \in \mathbb{R}^{4}: \xi_{\min } \leq \xi \leq \xi_{\max }\right\} \subset \mathbb{R}^{4}$ and $\hat{\mathcal{U}}=$ $\left\{u \in \mathbb{R}^{2}: u_{\min } \leq u \leq u_{\max }\right\} \subset \mathbb{R}^{2}$ are states and inputs polytope admissible regions (subscripts min and max are the minimum and maximum of the corresponding values). According to the system model 1 , the following cost function is formulated as follows:

$$
\begin{aligned}
\mathcal{J}\left(x(t), \mathcal{U}_{t}\right)= & \sum_{k=0}^{N_{p}}\left\|x_{t+k \mid t}-x_{\text {dest }+k \mid t}\right\|_{Q}^{2}+\sum_{k=0}^{N_{c}}\left\|u_{t+k \mid t}\right\|_{R}^{2} \\
& +\sum_{k=0}^{N_{c}}\left\|\varepsilon_{f_{t+k \mid t}}\right\|_{Y}^{2}+\left\|\varepsilon_{r_{t+k \mid t}}\right\|_{\Xi}^{2}
\end{aligned}
$$

where $\xi_{t+k \mid t}$ is the predicted state trajectory at time $t+k$ obtained by applying the control sequence $\mathcal{U}_{t}=$ $\left[u_{t}^{T}, \ldots, u_{t+N c-1}^{T}\right]^{T}$ to the system (1), starting from initial state of $\xi_{t \mid t} . N_{p} \in \mathbb{N}^{+}, N_{c} \in \mathbb{N}^{+}$, are prediction and control horizon, respectively where $N_{p} \geq N_{c} . Q \in$ $\mathbb{R}^{4 \times 4}, R \in \mathbb{R}^{2 \times 2}$ Are weighting matrices. $\varepsilon_{f_{t+k \mid t}}=$ $\left[\varepsilon_{x f k}, \varepsilon_{y f k}, \varepsilon_{f k}\right]^{T} \in \mathbb{R}^{3}$ and $\varepsilon_{r_{t+k \mid t}}=\left[\varepsilon_{x r k}, \varepsilon_{y r k}, \varepsilon_{r k}\right]^{T} \in \mathbb{R}^{3}$ are slack variables with $Y$ and $\Xi$ as their corresponding weight, for the softening the collision avoidance constraints. In (3), the first summation is the penalty on the reference tracking error, the second summation is a measure of the control effort, and the third one penalizes the violation of the collision avoidance constraints. The desired state $\xi_{\text {dest } t+k \mid t}$ representing reference signal that vehicle aims to follow at time $t+k$ and is defined as $\xi_{\text {des }}=\left[x_{\text {des }}, y_{\text {des }}, v_{x d e s}, 0\right]^{T}$, where $x_{\text {des }}, y_{\text {des }}$ are the reference target coordinates, and the desired velocity is selected as:

$$
v_{x, d e s}=\frac{\left\|x_{\text {des }}-x_{0}\right\|}{t^{*}}
$$

where, $t^{*}$ denotes a finite time horizon within which the controller needs to achieve the $v_{x, d e s}$. Consequently, the following constrained optimal control problem, for each sampling time, is formulated as:

$$
\min _{\mathcal{U}} \mathcal{J}\left(\xi(t), \mathcal{U}_{t}\right)
$$

subject to

$$
\begin{array}{cc}
\xi_{t+k+1}=A \xi_{t+k}+B u_{t+k} \\
\xi_{t+k} \in \hat{\mathcal{X}} \quad \mathrm{k}=1, \ldots, N_{p} \\
u_{t+k} \in \hat{\mathcal{U}} \quad \mathrm{k}=1, \ldots, N_{c}
\end{array}
$$

The control input is calculated as the state feedback law $u(x(t))=U_{t}^{*}$, which is solved in receding horizon framework [20] as a solution to the problem (5). In this framework, at every time step, the problem (5) and its constraints $((6)-(8))$ are calculated based on the current state $\xi(t)$, over a shifted time horizon. As the sets $\hat{\mathcal{X}}$ and $\hat{\mathcal{U}}$ are convex, then the MPC problem (5) is solved as a standard Quadratic Programming (QP) [21] optimisation problem. In order to avoid any potential collision, the safety constraints can be formulated as linear inequality constraints. Forward Collision Constraint (FCC), see Fig. 3. and Rear Collision Constraints (RCC), see Fig. 4, are two linear inequality constraints which are formulated as (9) and (10). The former represents the constraint in order to avoid collision between the subject vehicle and the preceding vehicle while the later indicates keeping safe distance when the subject vehicle tends to go back to its original lane.

$$
\begin{array}{r}
\frac{\Delta x_{k}}{L_{f}}-\frac{\Delta y_{k}}{W}+\eta \varepsilon_{x f k}+\frac{\varepsilon_{y f k}}{\varphi}+\varepsilon_{f k} \geq 1 \\
\frac{\Delta x_{k}}{L_{r}}+\frac{\Delta y_{k}}{W}-\eta \varepsilon_{x r k}-\frac{\varepsilon_{y r k}}{\varphi}+\varepsilon_{r k} \leq-1
\end{array}
$$

where $\Delta x_{k}=x_{p}-x$ with $x_{p}$ and $x$ denote the longitudinal position of preceding and subject vehicle, respectively, and $\Delta y_{k}=y_{p}-y$ with $y_{p}$ and $y$ indicate lateral position of preceding and subject vehicle, respectively. The parameter $L_{f}$ and $W$ is set as:

$$
\begin{array}{r}
L_{f}=v_{x} t_{f}+L_{c} \\
W=\frac{1}{2} W_{L}+W_{c}
\end{array}
$$

where $v_{x}$ is the subject vehicle velocity, $t_{f}$ is the time gap that subject vehicle approaches to preceding vehicle, $L_{c}$ and $W_{c}$ are length and width of preceding 
vehicle, and $W_{L}$ is the lane width. Likewise, in equation (10) the parameter $L_{r}$ indicates as:

$$
L_{r}=v_{x} t_{r}+L_{c}
$$

where $t_{r}$ is the time gap when subject vehicle tend to get back to its original lane. Moreover, $\eta$ and $\varphi_{f}$ are tuning parameters that can be referred in [14].

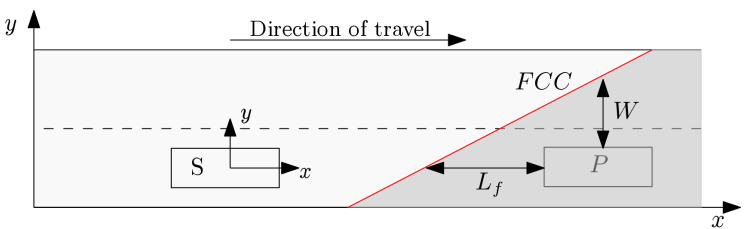

Fig. 3: Forward Collision Constraint, $\mathrm{S}$ and $\mathrm{P}$ stand for subject and preceding vehicle respectively

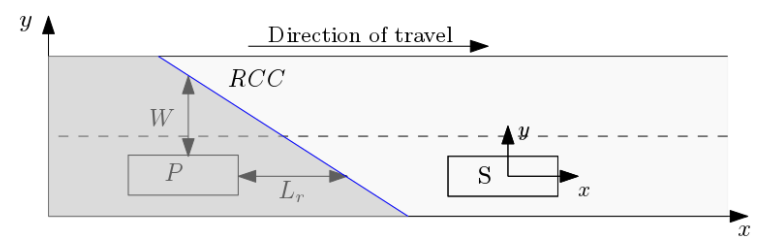

Fig. 4: Rear Collision Constraint, $\mathrm{S}$ and $\mathrm{P}$ stand for subject and preceding vehicle respectively

In Fig. 3, after identifying the preceding vehicle in front, the safety constraint, i.e. (9), is relaxed when subject vehicle crosses the preceding vehicle by tuning the gains Y. Subsequently in Fig. 4 after subject vehicle passed from preceding vehicle, the safety constraints (9) deactivates and RCC, i.e. (10) execute and relax when subject vehicle shift back to its original lane by tuning the weight $\Xi$.

\section{B. Torque Generator}

Once the desired speed from MPC is known, a feedback controller is formulated according to the error between the desired and actual vehicle speeds. In this paper, a proportional controller [22] is used in order to calculate the braking/traction torque which needs to be applied to the vehicle. The following equation represents the required torque which is equally distributed among the wheels:

$$
T=K_{p}\left(v_{x r e f}-v_{x}\right)
$$

where $K_{p}$ is the proportional gain. The generated torque $T$, following with calculated steering $\delta_{f}$ from MPC, will be fed to the CIM block to develop the desired CG forces, as well.

\section{Torque Vectoring Controller}

The desired C.G. forces derived from the CIM block are defined as:

$$
F_{d e s}=\left[F_{x}^{*}, F_{y}^{*}, G_{z}^{*}\right]^{T}
$$

where $F_{x}^{*}, F_{y}^{*}$ and $G_{z}^{*}$ are the desired C.G. longitudinal and lateral forces and yaw moment, respectively. The actual C.G. forces of the vehicle are:

$$
F=\left[F_{x}, F_{y}, G_{z}\right]^{T}
$$

where $F_{x}, F_{y}$ and $G_{z}$ are the actual C.G. longitudinal and lateral forces and yaw moment of the vehicle, respectively. The C.G. forces are function of the longitudinal and lateral tire forces (see Fig. 5) as:

$$
\begin{aligned}
F_{x} & =F_{x}\left(f_{x 1}, \ldots, f_{x 4}, f_{y 1}, \ldots, f_{y 4}\right) \\
F_{y} & =F_{y}\left(f_{x 1}, \ldots, f_{x 4}, f_{y 1}, \ldots, f_{y 4}\right) \\
G_{z} & =G_{z}\left(f_{x 1}, \ldots, f_{x 4}, f_{y 1}, \ldots, f_{y 4}\right)
\end{aligned}
$$

where, $f_{x i}$ and $f_{y i},(i=1, \ldots, 4)$ are longitudinal and lateral tire forces on each wheel of the vehicle. The corresponding adjusted C.G. forces to minimize the error between the actual $F$ and desired, $F_{d e s}$, is represented by:

$$
F(f+\delta f) \approx F(f)+\nabla F(f) \delta f
$$

where, $\nabla F(f)$ is the Jacobian matrix and $f$ is the total longitudinal and lateral forces on each wheel. The control action needed to minimize the error is:

$$
\delta f=\left[\delta f_{x 1}, \ldots, \delta f_{x 4}, \delta f_{y 1}, \ldots, \delta f_{y 4}\right]^{T}
$$

and is formulated as:

$$
\begin{gathered}
\delta f=\left[W_{d f}+W_{f}+\left(\nabla F(f)^{T} W_{E}\right) \nabla F(f)\right]^{-1} \\
.\left[\nabla F(f)^{T}\left(W_{E} E\right)-W_{f} f\right]
\end{gathered}
$$

Where $W_{d f}, W_{f}$ are weighing matrices. The applied corrective torque on each wheel is $\delta Q=R_{e f f} \times \delta f$. For brevity of the paper, further details about calculating control actions and weighting metrics are available in [15][23].

\section{Simulation Results}

In this section, a simulation environment is used to evaluate the effectiveness of the proposed integrated controller to perform evasive manoeuvres while the subject vehicle is traveling at high speeds and subjected to external disturbances. The vehicle parameters

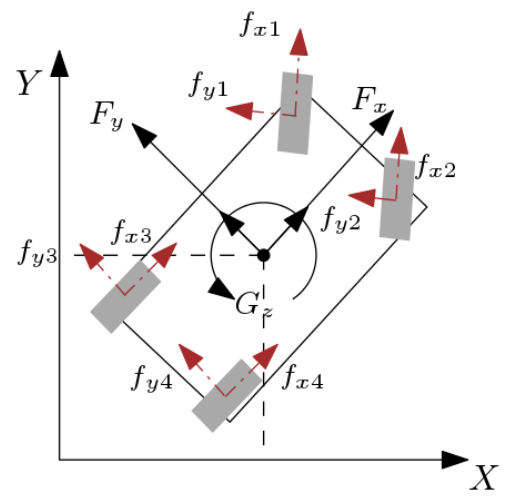

Fig. 5: Force conventions 
used in this simulation are tabulated in Table I] The simulation results for trajectory planning and torque vectoring controllers are conducted using combined IPG carmaker and MATLAB/Simulink software. The simulation is performed with the following parameters:

- $L_{c}=5 \mathrm{~m}, W_{c}=1.7 \mathrm{~m}, t_{f}=1.2 \mathrm{~s}, t_{r}=1.2 \mathrm{~s}, W_{L}=5 \mathrm{~m}$

- $N_{p}=20, N_{c}=4, T_{s}=0.1 \mathrm{~s}$ (sampling time), $t^{*}=$ $1.2 \mathrm{~s}$

- $Q=\operatorname{diag}\left(1 e^{4}, 1 e^{0}, 5 e^{-2}, 3 e^{1}\right), R=\operatorname{diag}\left(1 e^{3}, 1 e^{2}\right)$

- $-[0,0,0,15]^{T} \leq x \leq\left[1 e^{5}, 100,10,15\right]^{T}$

- $-[5,5]^{T} \leq u \leq[5,2]^{T}$

- $\mathrm{Y}=\left[1 e^{2}, 1 e^{5}, 1 e^{5}\right]^{T}, \Xi=\left[1 e^{-2}, 1 e^{-1}, 1 e^{-1}\right]^{T}$

- $W_{d f}=\operatorname{diag}\left(1 e^{2}, 1 e^{2}, 1 e^{2}, 1 e^{2}\right)$

- $W_{E}=\operatorname{diag}(0.4,0.02,1500)$

- $K_{p}=9 e^{3}$

The simulation is initialised with the subject vehicle travelling at $28 \mathrm{~m} \mathrm{~s}^{-1}$ and the preceding vehicle travelling at $20 \mathrm{~m} \mathrm{~s}^{-1}$. It is also assumed that both vehicles travel on a two-lane highway road with an initial distance of $80 \mathrm{~m}$ from each other. The simulation has been carried out assuming that the preceding vehicle performs a heavy braking action under low surface friction road conditions, i.e., road friction coefficient equal to 0.5 , with and without heavy crosswind. The speed of the preceding vehicle is shown in Fig. 6. It is noted that the preceding vehicle decreases its speed with slight braking action for $5 \mathrm{~s}$, and starts to perform a full braking action for the rest of the manoeuvre. It is noteworthy that for both cases the CIM has been tuned considering a nominal driving condition where the road friction coefficient is equal to one. Moreover,

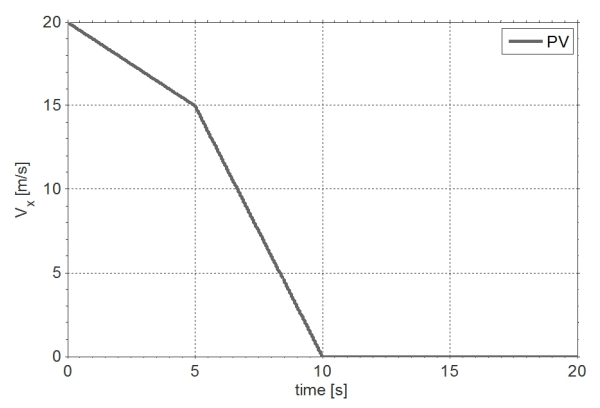

Fig. 6: Preceding vehicle velocity

in the rest of this section, it is noted that the path trajectory, $(x, y)$, is the optimal MPC trajectory and it represents the path that the vehicle should execute in nominal conditions. The path is obtained when the

TABLE I: Vehicle parameters

\begin{tabular}{ccc}
\hline Properties & Symbols & Value(Unit) \\
\hline Front distance from vehicle C.G & $l_{f}$ & $1.43 \mathrm{~m}$ \\
Rear distance from vehicle C.G & $l_{r}$ & $1.21 \mathrm{~m}$ \\
Vehicle mass & $M$ & $1360 \mathrm{Kg}$ \\
Yaw moment of inertia & $I_{z}$ & $2050 \mathrm{Kgm}^{2}$ \\
Effective wheel radius & $R_{e f f}$ & $0.3 \mathrm{~m}$ \\
\hline
\end{tabular}

optimal steering $\delta_{f}$, obtained from the MPC, is applied to the vehicle model in Fig. 2 In the case there is no cross-wind, the trajectory of the preceding vehicle, subject vehicle and the optimal MPC trajectory are shown in Fig. 7(a) while Fig. $7(b)$ depicts the position of the subject vehicle in the obstacle frame. Fig. 7(a) confirms that when the proposed control approach is used, the vehicle stays within the road boundaries while closely following the optimal MPC path trajectory despite the low-friction condition was not considered when computing the optimal steering. Moreover, it is noted that the vehicle starts to steer when it is $25 \mathrm{~m}$ from the preceding vehicle as shown in Fig. $7(\mathrm{~b})$. The closedloop dynamics of the system and the evolution of the system states are depicted in Fig. 8 Fig. 8 confirms that speed of the vehicle closely follows the reference speed while the inputs to the vehicle and input states are confined in their admissible sets. Moreover, after the harsh braking of the preceding vehicle (at $t=5 \mathrm{~s}$ ), the subject vehicle performs the following actions at 5.6s: (i) decreasing the longitudinal velocity of the subject vehicle, (ii) providing steering action to avoid the obstacle, (iii) providing maximum longitudinal deceleration in 0.5 friction. It is remarked that the stabilisation of the vehicle on the path, which allows the vehicle to successfully avoid the obstacle despite low friction, is guaranteed by the torque vectoring controller. In order to confirm the ability of the torque vectoring controller as an effective system during evasive manoeuvres, the architecture in Fig. 1 has been also tested when the torque vectoring system is excluded. The effectiveness of the torque vectoring as a stabilization controller is presented in Fig. 9 where the path executed by the vehicle as well as the heading angle are depicted with and without the torque vectoring system. The top figure in Fig. 9 shows that the vehicle without torque vectoring controller fails to perform the evasive manoeuvring. This is also confirmed by the bottom figure in Fig. 9 where, the uncontrolled vehicle violates the yaw angle constraints, leading the vehicle to spin. On the other
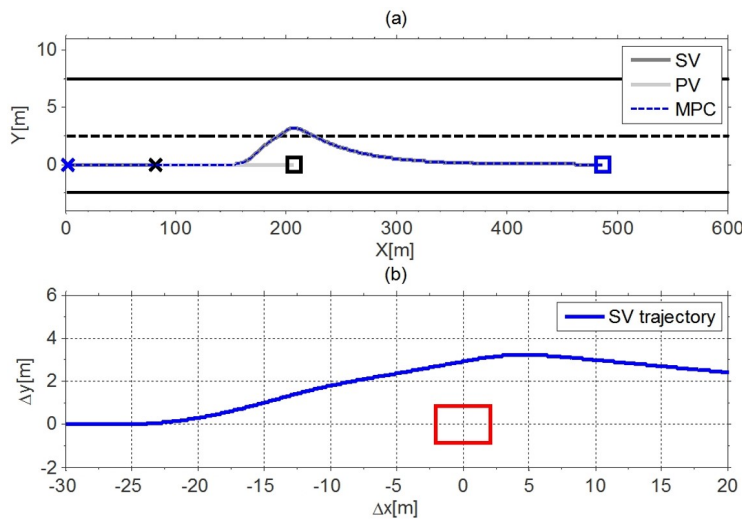

Fig. 7: Subject vehicle $(S V)$ and preceding vehicle $(P V)$ trajectories 
(a)

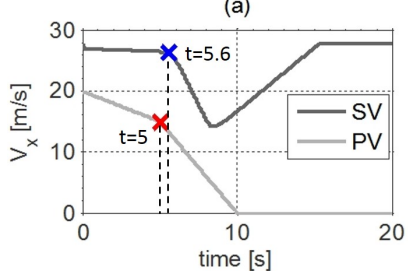

(c)

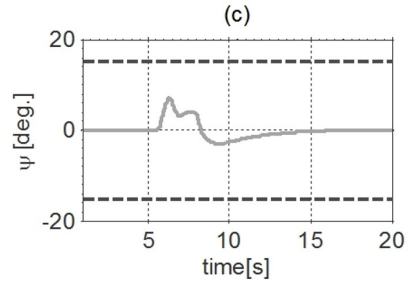

(b)

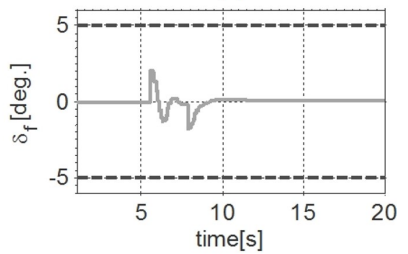

(d)

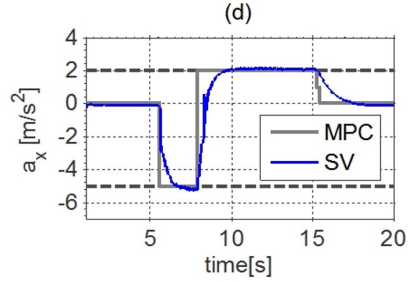

Fig. 8: Simulation results: Subject vehicle $(S V)$ and preceding vehicle $(P V)$ (a) longitudinal velocity, (b) steering angle, (c) heading angle and (d) longitudinal acceleration. (--) represent the system constraints

hand, the controlled vehicle, significantly improves the manoeuvrability and stability of the vehicle in case of low friction surface. An in-depth comparison of the closed-loop system behaviour with and without the torque vectoring system is reported in Fig. 10 which shows the errors of CG forces (i.e., $e$ in Fig. 1 ) are compared with and without torque vectoring control. It is noted that the for both cases the CIM provides the nominal reference C.G. forces when the road friction condition is one. In this figure, the errors are significantly damped compare to the vehicle without torque vectoring controller, resulting in a superior vehicle handling performance. However, it is noteworthy to point out that, since there is no direct control of lateral tire forces the magnitude of the error in lateral CG force in Fig. 10(b) is relatively large in time interval between $6 s$ to $9 \mathrm{~s}$. The applied control action from the torque vectoring control and overall torque adjustment at each wheel are shown in Fig. 11(a) and Fig. 11(b) where FR, FL, RR and RL indicate the front Front Left, Front Right, Rear Left and Rear Right wheel, respectively. As can be seen from these plots, the distribution of the torque is symmetric and torque vectoring control aims to generate the corrective yaw moment to stabilize the vehicle. Moreover, at 5.6s, the torque generated from torque vectoring control on the FR, FL, RR and RL wheels are $25,-25,25,-25$ N.m, respectively. These values show that the vehicle is negotiating a left turn. The total torque provided to the vehicle is shown in Fig. 11(b).

For the second scenario, the crosswind is considered together with an unmodelled low friction condition $(\mu=0.5)$, when the preceding vehicle velocity is that the same as in Fig. 6. It is noted that cross wind can be considered as a disturbance on highways which intend to diverge the vehicle from its desired trajectory

and increases the probability of an accident. In the simulation, a gust of wind of $120 \mathrm{~km} / \mathrm{h}$ at 90 degree angle in reference to the road, has been considered during the entire execution of the evasive manoeuvre.

The path executed by the vehicle with and without the torque vectoring stabilisation system is shown in Fig. 12 where shaded blue rectangle represents the region where the wind disturbance is active. Fig. 12(a) confirms the capability of the integrated control system to safely avoid collisions also in such a challenging emergency scenario and despite the vehicle is pushed by the severe wind force. The proposed integrated controller keeps the vehicle in the intended path and maintains the directional stability of the vehicle (see also Fig. 12(b) where the heading of the vehicle is depicted), while the vehicle without torque vectoring controller, fails to complete the evasive manoeuvring. As expected, in order to stabilize the vehicle while keeping it in the lane limits despite of heavy crosswind, the closed-loop torque vectoring controller generates higher correcting toque control actions with respect to the case there is no wind in order to compensate the presence of the external disturbance. This is confirmed in Fig. 13 . For the simulation purposes, C.G. error forces for controlled and uncontrolled vehicle under heavy crosswind are compared in Fig. 14(a-c). These errors converge to zero as control actions are applied on each wheel, resulting in better handling and manoeuvrability under low surface friction $(\mu=0.5)$ and heavy crosswind. However, as expected, the lateral C.G. error is relatively high compare to longitudinal and yaw moment errors. This is due to the fact that there is no direct adjustment on lateral control force and only longitudinal force is taking in to account as
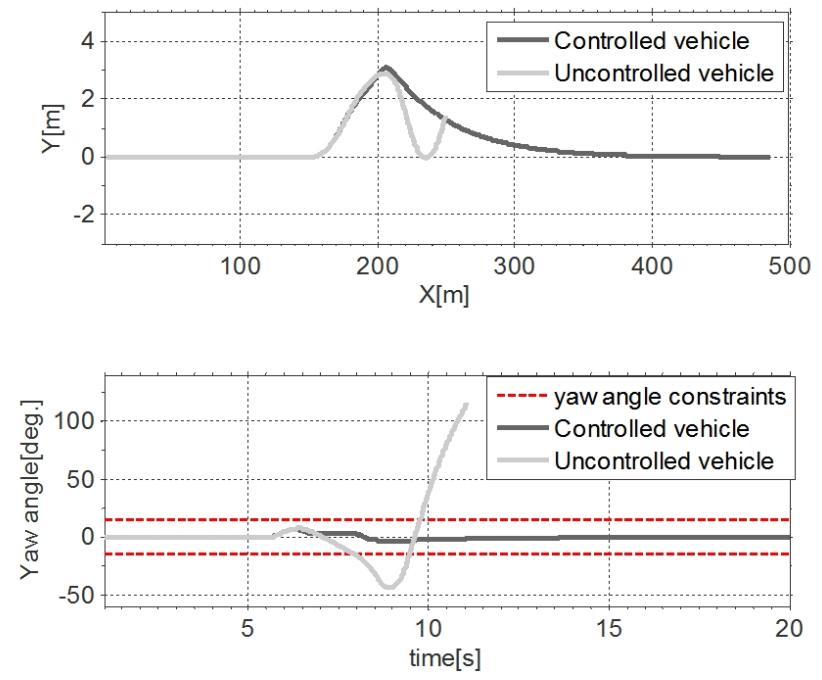

Fig. 9: Trajectory and yaw angle comparison between controlled and uncontrolled vehicle 

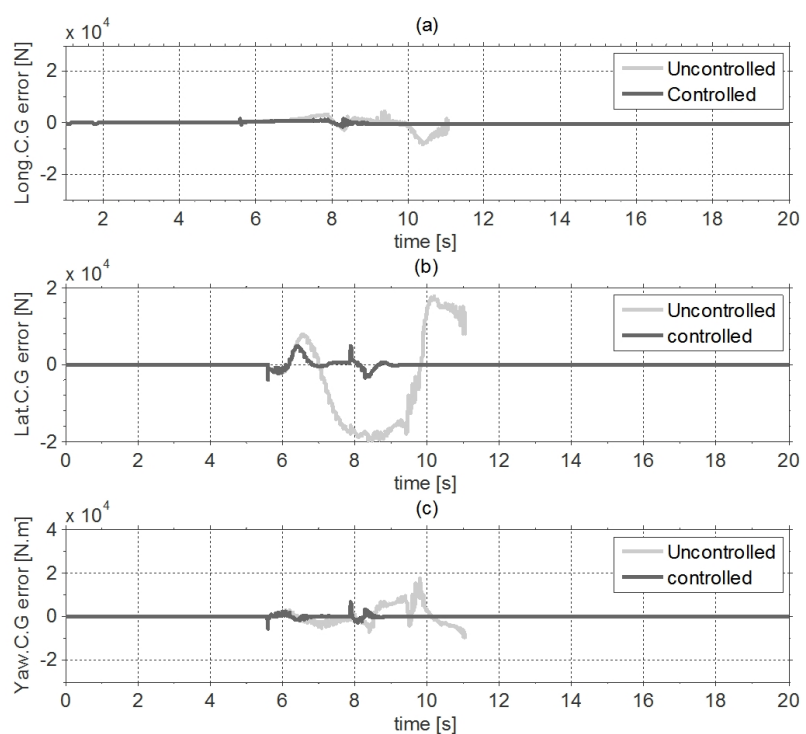

Fig. 10: C.G. error forces with $\mu=0.5$

(a)

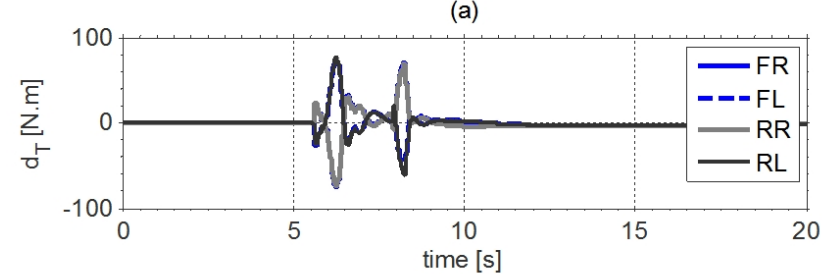

(b)

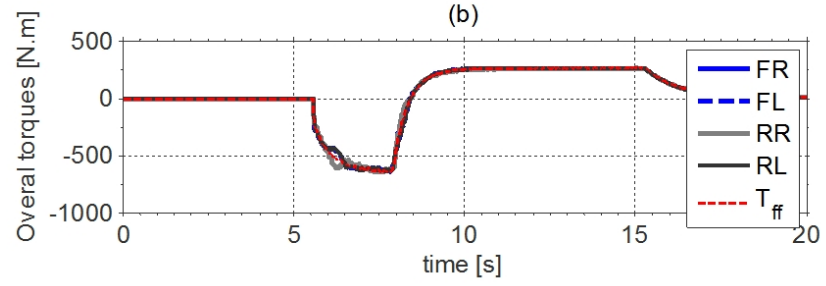

Fig. 11: Control action and overall torque adjustment

control effort as previously was discussed.

Finally, a parametric analysis has been carried out to verify the effectiveness of the proposed integrated control framework. In this analysis, the maximum error between lateral displacement (Fig. 15(a)) and heading angle (Fig. 15(b)) from trajectory planner and the actual vehicle response under heavy crosswind and different road conditions has been tested for controlled and uncontrolled vehicle. The lateral displacement error $\left(e_{y}\right)$ for controlled vehicle is increasing gradually with lowering friction surface. Although this plot demonstrates a peak deviation of approximately $0.59 \mathrm{~m}$, the proposed integrated controller maintain the vehicle from further rise of deviation, ensuring the vehicle remains within the lane limits. Whereas the uncontrolled vehicle with nearly $2 \mathrm{~m}$ deviation starts to veer off from the desired path (Fig. 15(a)). Similarly, for heading angle error

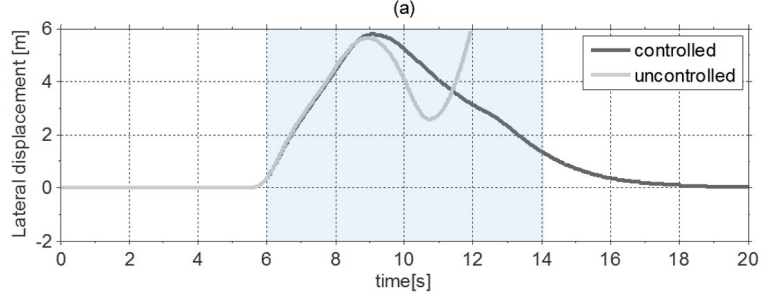

(b)

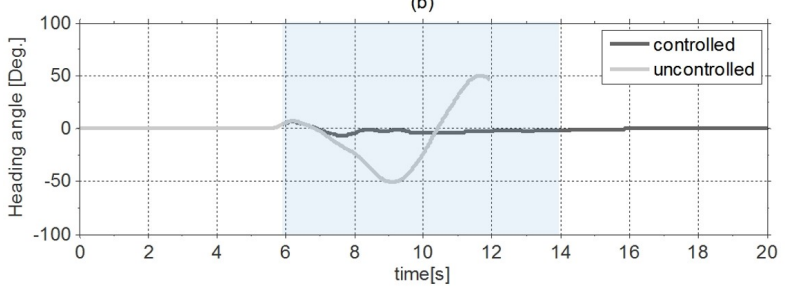

Fig. 12: Lateral displacement and heading angle under severe cross wind

(a)
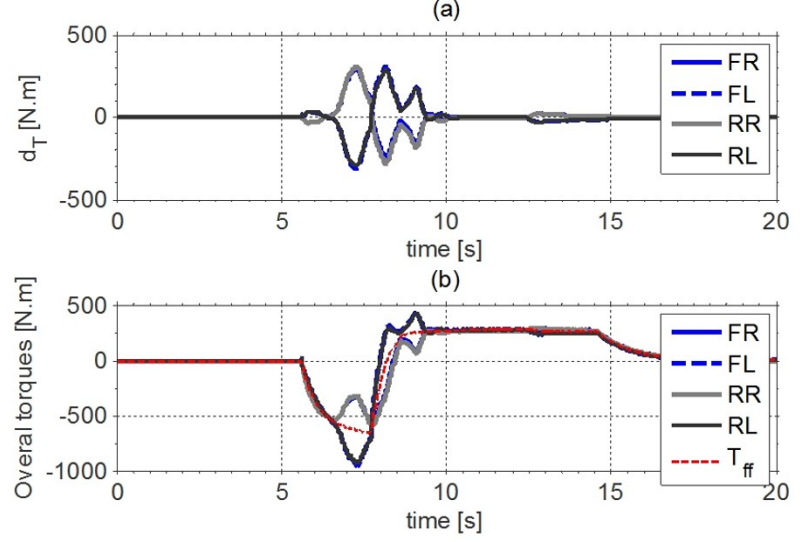

Fig. 13: Adjusted torque on each wheel

$\left(e_{\psi}\right)$, the maximum deviation for uncontrolled vehicle is approximately 70 degrees, meaning that the vehicle starts to spin and fails to maintain its stability, while the controlled vehicle heading error reaches to about 3.9 degree (Fig. 15(b)). This analysis demonstrates the effectiveness of the integrated controller for vehicle stabilization while avoiding collision under different values of friction and heavy crosswind.

\section{CONCLUSIONS}

An integrated control algorithm for performing evasive manoeuvres at highway speeds under model uncertainty and external disturbances was presented in this paper. The proposed control framework consists of (i) trajectory planning controller for designing collisionfree trajectories and (ii) a torque vectoring controller to ensure lateral-yaw stability of the vehicle. The closedloop scheme was implemented on an IPG CarmakerMATLAB Co-simulation platform and numerical results demonstrated the benefits of the proposed controllers. 

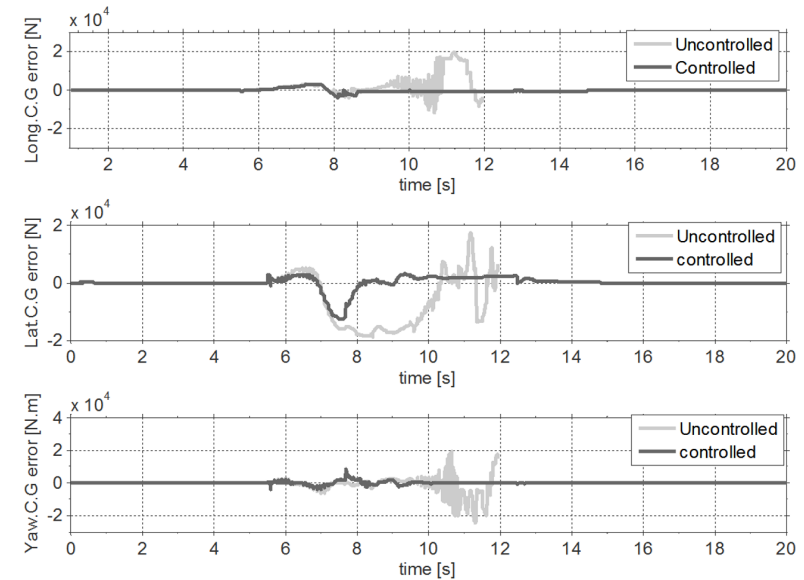

Fig. 14: C.G errors under severe gust of wind

(a)

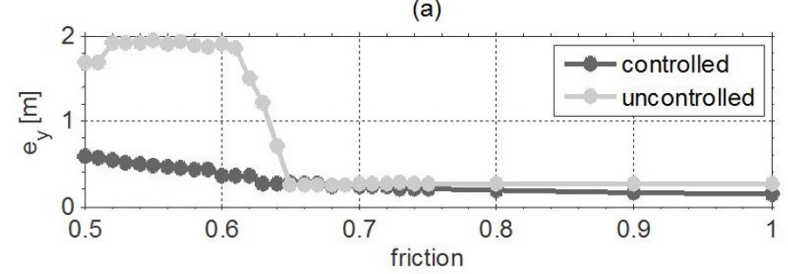

(b)

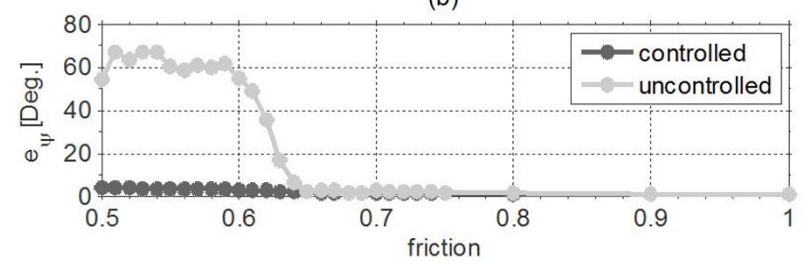

Fig. 15: Lateral displacement and heading angle error

The trajectory planning performed using an MPC controller computed feasible and collision-free trajectories for evasive manoeuvres at high-speeds. Moreover, a torque vectoring controller designed using optimal control technique was utilised to ensure that the vehicle followed the reference trajectory without loss of stability while subjected to external disturbances such as (i) low friction, (ii) crosswinds, and (iii) changes in obstacle velocity. Additionally, a parametric analysis illustrated the robustness of the proposed integrated control framework for performing high-speed evasive manoeuvres safely under a wide range of road friction conditions.

\section{REFERENCES}

[1] A.Sorniotti, P.Barber, and S. Pinto, "Automated Driving," in Automated Driving, 2017, pp. 71-140.

[2] S. Dixit, U. Montanaro, S. Fallah, M. Dianati, D. Oxtoby, T. Mizutani, and A. Mouzakitis, "Trajectory Planning for Autonomous High-Speed Overtaking using MPC with Terminal Set Constraints," in 2018 21st International Conference on Intelligent Transportation Systems (ITSC), 2018, pp. 1061-1068.

[3] B. Liu, Q. Shi, Z. Song, and A. El, "Simulation Modelling Practice and Theory Trajectory planning for autonomous intersection management of connected vehicles," Simulation Modelling Practice and Theory, vol. 90, no. October 2018, pp. 16-30, 2019.
[4] R. Schmied and P. Colaneri, "Mixed $\mathrm{H}_{2}-\mathrm{H}_{\infty}$ control for automated highway driving," Mechatronics, vol. 57, no. June 2017, pp. 63-72, 2019.

[5] K. Reif, Brakes, Brake Control and Driver Assistance Systems, 2014

[6] T. Shim, G. Adireddy, and H. Yuan, "Autonomous vehicle collision avoidance system using path planning and modelpredictive-control-based active front steering and wheel torque control," vol. 226, no. 6, pp. 767-778, 2012.

[7] J. Shah, M. Best, and A. Benmimoun, "Autonomous rear-end collision avoidance using an electric power steering system," vol. 229, no. 12, pp. 1638-1655, 2015.

[8] Y. Gao, T. Lin, F. Borrelli, E. Tseng, and D. Hrovat, "Predictive Control of Autonomous Ground Vehicles With Obstacle Avoidance on Slippery Roads," ASME 2010 Dynamic Systems and Control Conference, Volume 1, pp. 265-272, 2010.

[9] M. Cao, R. Wang, J. Wang, and N. Chen, "An Integrated MPC Approach for FWIA Autonomous Ground Vehicles with Emergency Collision Avoidance," pp. 2432-2437, 2018.

[10] J. Funke, M. Brown, S. M. Erlien, and J. C. Gerdes, "Collision Avoidance and Stabilization for Autonomous Vehicles in Emergency Scenarios," IEEE Transactions on Control Systems Technology, vol. 25, no. 4, pp. 1204-1216, 2017.

[11] C. Chatzikomis, A. Sorniotti, P. Gruber, M. Zanchetta, and D. Willans, "Comparison of Path Tracking and Torque- Vectoring Controllers for Autonomous Electric Vehicles," IEEE.

[12] L. D. Novellis, A. Sorniotti, P. Gruber, J. Orus, J.-m. R. Fortun, J. Theunissen, and J. D. Smet, "Direct yaw moment control actuated through electric drivetrains and friction brakes : Theoretical design and experimental assessment," Mechatronics, vol. 26, pp. $1-15,2015$

[13] T. Goggia, A. Sorniotti, L. D. Novellis, A. Ferrara, S. Member, P. Gruber, J. Theunissen, D. Steenbeke, B. Knauder, and J. Zehetner, "Integral Sliding Mode for the Torque-Vectoring Control of Fully Electric Vehicles : Theoretical Design and Experimental Assessment," IEEE Transactions on Vehicular Technology, vol. 64, no. 5, pp. 1701-1715, 2015.

[14] J. Nilsson, P. Falcone, M. Ali, and J. Sjöberg, "Receding horizon maneuver generation for automated highway driving," Control Engineering Practice, vol. 41, pp. 124-133, 2015.

[15] S. Fallah, A. Khajepour, B. Fidan, S.-K. Chen, and B. Litkouhi, "Vehicle Optimal Torque Vectoring Using State-Derivative Feedback and Linear Matrix Inequality," vol. 62, no. 4, pp. 1540-1552, 2013.

[16] P. Falcone, M. Tufo, F. Borrelli, J. Asgarit, and H. E. Tseng, "A linear time varying model predictive control approach to the integrated vehicle dynamics control problem in autonomous systems," Proceedings of the IEEE Conference on Decision and Control, pp. 2980-2985, 2007.

[17] H. B. Pacejka, Tire Characteristics and Vehicle Handling and Stability, 2012.

[18] THE EUROPEAN COMMISSION, “COMMISSION REGULATION (EU) No 347/2012," pp. 1-17, 2012.

[19] J. Kong, M. Pfeiffer, G. Schildbach, and F. Borrelli, "Kinematic and Dynamic Vehicle Models for Autonomous Driving Control Design," 2015 IEEE Intelligent Vehicles Symposium (IV), no. Iv, pp. 1094-1099, 2015

[20] B. D. Schutter, J. Ploeg, L. D. Baskar, and H. Nijmeijer, Hierarchical, Intelligent and Automatic Controls, 2012, no. July 2015.

[21] J. Nilsson, Y. Gao, A. Carvalho, and F. Borrelli, "Manoeuvre generation and control for automated highway driving," IFAC Proceedings Volumes, vol. 47, no. 3, pp. 6301-6306, 2014.

[22] K. Jalali, S. Lambert, and J. Mcphee, "Development of a Pathfollowing and a Speed Control Driver Model for an Electric Vehicle," 2017.

[23] S.-k. Chen, S.-k. Chen, Y. Ghoneim, N. Moshchuk, and B. Litkouhi, "Tire Force-based Holistic Corner Control," no. November 2015, 2012. 